\title{
Factor Graph Weight Particles aided Distributed Underwater Cooperative Positioning Algorithm
}

\section{lingling zhang}

Northwestern Polytechnical University

baoguo yu

the 54th reasearch institute of china electronics technology group corporation

Chengkai Tang ( $\nabla$ tangchengkai99@163.com )

Northwestern Polytechnical University https://orcid.org/0000-0002-9008-0061

yi zhang

Northwestern Polytechnical University

Houbing Song

Embry-Riddle Aeronautical University

\section{Research Article}

Keywords: Distributed co-localization, underwater node, factor graph, weighted particle

Posted Date: April 26th, 2021

DOI: https://doi.org/10.21203/rs.3.rs-345295/v1

License: (c) (i) This work is licensed under a Creative Commons Attribution 4.0 International License. Read Full License 


\title{
Factor Graph Weight Particles aided Distributed Underwater Cooperative Positioning Algorithm
}

\author{
Lingling Zhang ${ }^{1} \cdot$ Baoguo $\mathrm{Yu}^{2 *}$. Chengkai Tang ${ }^{3 *} \cdot \mathrm{Yi} \mathrm{Zhang}^{3}$. \\ Houbing Song ${ }^{4}$
}

Received: date / Accepted: date

\begin{abstract}
The growing scale of marine exploration requires high-resolution underwater localization, which necessitates cooperation among underwater network nodes, considering the channel complexity and power efficiency. In this paper, we proposed factor graph weight particles aided distributed underwater nodes cooperative positioning algorithm (WP-DUCP). It capitalized on the factor graph and sum-product algorithm to decompose the global optimization to the product of several local optimization functions. Combined with the Gaussian parameters to construct the weighted par-
\end{abstract}

Lingling Zhang

E-mail: llzhang@nwpu.edu.cn

Baoguo Yu

E-mail: yubg@sina.cn

$\square$ Chengkai Tang

E-mail: cktang@nwpu.edu.cn

Yi Zhang

E-mail: zhangyi@nwpu.com

Houbing Song

E-mail: Houbing.Song@erau.edu

corresponding author:Chengkai Tang

E-mail: cktang@nwpu.edu.cn

1 School of Marine Science and Technology, Northwestern Polytechnical University, Xi'an, China 710072

2 The 54th Research Institute of China Electronics Technology Group Corporation, Shijiazhuang, China

3 School of Electronics and Information, Northwestern Polytechnical University, Xi'an, China 710072

4 Department of Electrical, Computer, Software, and Systems Engineering, Embry-Riddle Aeronautical University, FL, USA 32114 ticles and to realize the belief transfer, it shows low complexity and high efficiency, suitable to the energyrestricted and communication distance-limited underwater networks. In terms of convergence, localization resolution, and computation complexity, we conducted the simulation and real-test with comparison to the existing co-localization methods. The results verified a higher resolution of the proposed method with no extra computation burden.

Keywords Distributed co-localization · underwater node $\cdot$ factor graph $\cdot$ weighted particle

\section{Introduction}

With the large-scale utilization of Marine resources, underwater activities have shifted from the military field to the civil-military integration and civilian-oriented development [1]. With the application of fishery monitoring, ocean current monitoring, oil drilling platform, and other underwater systems, underwater node positioning is becoming more critical[2]. Most existing underwater positioning capitalized on the GNSS aided floating buoy and water pressure meter. However, its impractical to deploy a buoy positioning system for every underwater node[3]. To this end, the research underwater co-localization based on the high precision underwater node is undergoing recently. Earlier underwater co-localization draws lessons from terrestrial wireless co-localization systems[4]. The co-localization method based on minimum mean squared error(MMSE) is proposed in [5]. The co-localization method based on maximum a posteriori(MAP) is presented in [6]. These two methods adopted the marginal probability density function(PDF) of the underwater node position. However, the lack of high ranging precision in underwater 
systems degraded the co-localization performance. The co-localization method based on the factor graph is proposed in [7]. It mapped the statistical graph model into network topology and obtained the probability function through message passing over the network. If enough nodes exist, it will provide high localization precision. However, this centralized processing increased the computation burden and the communication transmission requirement. A mixed distributed message passing algorithm based on belief propagation and mean field(MF) is proposed in [8]. It adopted the belief propagation in the motion-related, while adopted the mean field message passing in the measurement-related parts of the factor graph. And it further adopted the Gaussian approximation to decrease the computation burden. The co-localization method based on connectivity information-aided belief propagation(CIBP) is proposed in [9]. To improve the positioning accuracy, the semidefinite programming (SDP) method base on CIBP is proposed in [10]. Those methods integrated the connectivity information into the belief propagation and excluded the fake position to avoid the wrong message passing. The distributed co-localization method based on variational message passing (VMP) is proposed in [11]. It adopted second-order Taylor expansion to model the non-linear ranging function, then obtain the colocation with mean and covariance information. These above methods hold the hypothesis that the precision of ranging is higher than self-positioning. Its hard to ensure in the underwater environment. A Taylor expansion based distributed positioning (Taylor-DP) method is proposed in [12]. However, in the process of Taylor expansion, the high-order terms are omitted, which leads to low precision of cooperative positioning. In this paper, we proposed a distributed co-localization method based on weighted particles. It expresses the belief information with weighted particles and applies the sumproduct theory to pass the message within the factor graph.

\section{Underwater Co-localization Model}

Due to the ocean currents, the position of the underwater nodes are uncertain[13]. By the aid of hydraulic pressure meter, we model the underwater collaborative positioning system into two-dimensional planar, as shown in figure 1.

Suppose in this underwater co-localization network, there are $M$ anchor nodes(positions known, ensured by high precision positioning) and $N$ undetermined nodes (positions unknown). Denote the set of anchor nodes as $\mathcal{M}$, and the set of undetermined nodes as $\mathcal{N}$. Denote the set of all nodes in the network as $\mathcal{S}$, then we have $\mathcal{S}=$
$\mathcal{M} \cup \mathcal{N}$. Denote the position of node $i$ as $\mathbf{x}_{i}=\left[x_{i}, y_{i}\right]$, and the positions of all nodes in $\mathbf{S}$ as $\mathbf{X}=\left\{\mathbf{x}_{i} \mid i \in \mathcal{S}\right\}$. Denote the neighbour anchor nodes set of node $i$ as $\mathcal{M}_{i}$, and the neighbour undetermined nodes set of node $i$ as $\mathbf{N}_{i}$. Denote the set of all neighbour nodes of node $i$ as $\mathcal{S}_{i}$, then we have $\mathcal{S}_{i}=\mathcal{M}_{i} \cup \mathcal{N}_{i}$.

In this paper, we make the following assumptions:

1.The communication range and the measurement distance are equal, denote as $R$.

2.The ranging error of node $i$ and node $j$ follows the Gaussian distribution, denote as $n_{j->i} N\left(0, \sigma_{i j}^{2}\right)$

3 .The prior probability distribution of all underwater nodes are independence, and the prior probability distribution along $\mathrm{x}$-axis and $\mathrm{y}$-axis of each node are independence.

Under these assumptions, the range measurement between node $i$ to node $j \in \mathcal{S}_{i}$ can be expressed as

$z_{j->i}=\left\|\mathbf{x}_{j}-\mathbf{x}_{i}\right\|+n_{j->i}$

Where $\|\cdot\|$ denotes the Euclidean Norm. Denote $\mathcal{Z}_{i}=$ $\left\{z_{j->i} \mid j \in \mathcal{S}_{i}\right\}$, i.e. the set of range measurements from node $i$ to all the corresponding neighbour nodes. Denote $\mathcal{Z}=\left\{\mathcal{Z}_{i} \mid i \in \mathcal{N}\right\}$, i.e. the set of range measurements of all undetermined nodes to its corresponding neighbour nodes. The probability of the range measurement between node $i$ at position $\mathbf{x}_{i}$ to node $j$ at position $x_{j}$ is expressed as

$\left.p\left(z_{j->i} \mid \mathbf{x}_{i}, \mathbf{x}_{j}\right)=\frac{1}{\sqrt{2 \pi \sigma_{i j}^{2}}} \exp \left\{-\frac{z_{j->i}-\left\|\mathbf{x}_{j}-\mathbf{x}_{i}\right\|}{2 \sigma_{i j}^{2}}\right\} 2\right)$

According to Bayers estimation based on MMSE[14], the position estimation of the undetermined node $i$, i.e. $\mathbf{x}_{i}$, can be expressed through the posterior $\operatorname{PDF} p\left(\mathbf{x}_{i} \mid \mathcal{Z}\right)$

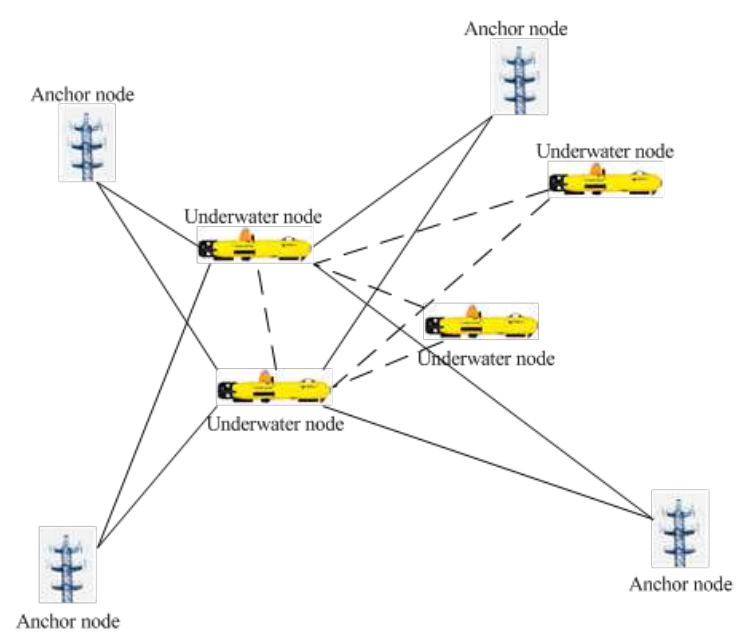

Fig. 1 Underwater Co-localization Model 
as

$\hat{\mathbf{x}}_{i}=\int \mathbf{x}_{i} p\left(\mathbf{x}_{i} \mid \mathcal{Z}\right) d \mathbf{x}_{i}$

the posterior PDF $p\left(\mathbf{x}_{i} \mid \mathcal{Z}\right)$ can be expressed as the marginal joint PDF as

$p\left(\mathbf{x}_{i} \mid \mathcal{Z}\right)=\int p(\mathbf{X} \mid \mathcal{Z}) d \mathbf{X} \backslash \mathbf{x}_{i}$

Where the slash in the $d \mathbf{X} \backslash \mathbf{x}_{i}$ is the setminus operator. According to the independence assumption we made earlier, we can express the joint PDF as

$$
\begin{aligned}
p(\mathbf{X} \mid \mathcal{Z}) & \propto p(\mathcal{Z} \mid \mathbf{X}) p(\mathbf{X}) \\
& =\prod_{i \in \mathcal{N}} \prod_{j \in \mathcal{S}_{i}} \prod_{a \in \mathcal{M}} p\left(\mathbf{x}_{i}\right) p\left(\mathbf{x}_{a}\right) p\left(z_{j->i} \mid \mathbf{x}_{i}, \mathbf{x}_{j}\right)
\end{aligned}
$$

Where $p\left(\mathbf{x}_{i}\right)$ and $p\left(\mathbf{x}_{a}\right)$ denote the PDF of undetermined node $i$ and anchor node $a$.

\section{Underwater Co-localization Method based on Weighted Particle}

Due to the high computational complexity and communication cost of the existing underwater cooperative positioning technology, the updating of node location is quite slow[15-17], and it is difficult to alleviate the error accumulation. To reduce the computation complexity and realize the rapid update of the node location, we constructed the corresponding factor graph and applied the sum-product theory for message passing. In this factor graph, the local information is expressed with weighted particles, and the belief is represented with Gaussian approximation. In this way, the belief of the undetermined node $i$, i.e. the posterior of the position $\mathbf{x}_{i}$, is expressed as

$$
b^{(l)}\left(\mathbf{x}_{i}\right) \propto f\left(\mathbf{x}_{i}\right) \prod_{i \in \mathcal{S}_{i}} m_{j->i}^{(l)}\left(\mathbf{x}_{i}\right)
$$

Where $l$ denotes the iterative index, $f\left(\mathbf{x}_{i}\right)$ denotes the prior PDF of position $\mathbf{x}_{i}$, and $m_{j->i}^{(l)}\left(\mathbf{x}_{i}\right)$ denotes the message passing from node $j$ to node $i$. According to the sum-product theory, the message is proportion to the likelihood function, prior PDF of neighbor node $j$ and the prod of message at the node $j$, as shown in Equation (7)

$$
\begin{aligned}
m_{j->i}^{(l)}\left(\mathbf{x}_{i}\right) & \propto \int p\left(z_{j->i} \mid \mathbf{x}_{i}, \mathbf{x}_{j}\right) f\left(\mathbf{x}_{j}\right) \prod_{k \in \mathcal{S}_{j} \backslash i} m_{k->j}^{(l)}\left(\mathbf{x}_{j}\right) d \mathbf{x}_{j} \\
& \propto \int p\left(z_{j->i} \mid \mathbf{x}_{i}, \mathbf{x}_{j}\right) \frac{b^{l-1}\left(\mathbf{x}_{j}\right)}{m_{i->j}^{(l)}\left(\mathbf{x}_{j}\right)} d \mathbf{x}_{j}
\end{aligned}
$$

Equation (7) shows that to get the belief in Equation (6), we need different message passing from each node to its neighbour nodes. It will definitely increase the communication cost. And also, the calculation of $m_{i->j}^{(l)}\left(\mathbf{x}_{j}\right)$ increased the complexity. Then we simply Equation (7) as

$m_{j->i}^{(l)}\left(\mathbf{x}_{i}\right) \propto \int p\left(z_{j->i} \mid \mathbf{x}_{i}, \mathbf{x}_{j}\right) b^{l-1}\left(\mathbf{x}_{j}\right) d \mathbf{x}_{j}$

For the anchor nodes at known position and undetermined nodes at unknown position, we adopt Gaussian Approximation to express the belief information as

$b^{(l)}\left(\mathbf{x}_{j}\right)=\left\{\begin{array}{cc}\delta\left(\mathbf{x}_{j}-\boldsymbol{\mu}_{j}\right) & j \in \mathbf{M}_{i} \\ N\left(\mathbf{x}_{j}, \boldsymbol{\mu}_{j}, \boldsymbol{\Sigma}_{j}\right) & j \in \mathbf{N}_{i}\end{array}\right.$

Substitute Equation (9) into Equation (8), we have

$m_{j->i}^{(l)}\left(\mathbf{x}_{i}\right) \propto \int p\left(z_{j->i} \mid \mathbf{x}_{i}, \mathbf{x}_{j}\right) N\left(\mathbf{x}_{j}, \boldsymbol{\mu}_{j}, \boldsymbol{\Sigma}_{j}\right) d \mathbf{x}_{j}$

It's difficult to calculate this integral because of the nonlinearity, so we expand the range information by first-order Taylor series as

$$
\begin{array}{r}
\left\|\mathbf{x}_{j}-\mathbf{x}_{i}\right\|=\left\|\hat{\mathbf{x}}_{j->i}\right\|-\frac{\hat{\mathbf{x}}_{j->i}}{\left\|\hat{\mathbf{x}}_{j->i}\right\|}\left(\mathbf{x}_{j}-\boldsymbol{\mu}_{j}\right)+ \\
\frac{\hat{\mathbf{x}}_{j->i}}{\left\|\hat{\mathbf{x}}_{j->i}\right\|}\left(\mathbf{x}_{i}-\hat{\mathbf{x}}_{i}\right)
\end{array}
$$

where $\hat{\mathbf{x}}_{j->i}=\hat{\mathbf{x}}_{i}-\boldsymbol{\mu}_{j}$. Substitute (12) to (10), we have

$$
\begin{aligned}
& m_{j->i}^{(l)}\left(\mathbf{x}_{i}\right) \propto \exp [-\frac{1}{2}\left(\hat{\mathbf{x}}_{j->i}-z_{j->i} \frac{\hat{\mathbf{x}}_{j->i}}{\left\|\hat{\mathbf{x}}_{j->i}\right\|}\right)^{T} \\
&\left.\Sigma_{j+z}^{-1}\left(\hat{\mathbf{x}}_{j->i}-z_{j->i} \frac{\hat{\mathbf{x}}_{j->i}}{\left\|\hat{\mathbf{x}}_{j->i}\right\|}\right)\right]
\end{aligned}
$$

where $\Sigma_{j+z}$ and $I$ denotes the unit matrix.

The weighted particle of node $i$ is expressed as

$$
W_{i}[n]=w_{i}[n] \prod_{j \in \mathcal{S}_{i}} m_{j->i}\left(\mathbf{x}_{i}[n]\right)
$$

Where $w_{i}[n]$ denotes the weight satisfies $\sum_{n=1}^{K} W_{i}[n]=$ 1 , and $K$ denotes the number of particles.

The communication cost in belief propagation relies on the expression of belief information. Most existing methods adopt the broadcasting method includ(7) ing all weighted particles, attribute to the high cost within multiple iterations. When the belief is irregular 
Table 1 algorithmic representation of WP-DUCP

$1 \quad$ Initialization

A for all $i \in \mathcal{S}$, run in parallel

(a1) prior information

$f\left(\mathbf{x}_{i}\right)=\left\{\begin{array}{cc}\delta\left(\mathbf{x}_{i}-\boldsymbol{\mu}_{i}\right) & i \in \mathbf{M} \\ N\left(\mathbf{x}_{i}, \boldsymbol{\mu}_{i}^{(0)}, \boldsymbol{\Sigma}_{i}(0)\right) & i \in \mathbf{N}\end{array}\right.$

Generate $\mathrm{K}$ particles satisfies the norm condition.

(a2) broadcasting information

node position $\boldsymbol{\mu}_{i}$, for $i \in \mathcal{M}$

mean $\boldsymbol{\mu}_{i}^{(0)}$ and variance $\boldsymbol{\Sigma}_{i}^{(0)}$ of node position, for $i \in \mathcal{N}$

B for all $i \in \mathcal{N}$, run in parallel

(b1) receive information $\sigma_{i->j}^{2}$ from neighbour nodes, $j \in \mathcal{S}_{i}$

(b2) measure the distance $z_{j->i}$ to neighbour nodes, $j \in \mathcal{S}_{i}$

$2 \quad$ Iteration

C for all $i \in \mathcal{N}$

(c1) receive information from anchor nodes $j \in \mathcal{M}_{i}($ Eq. 10)

(c2) receive information from other coordination $\operatorname{nodes}\left(\right.$ Eq. 14) $j \in \mathcal{N}_{i}$

(c3) update the weights (Eq. 15)

(c4) update the mean $\boldsymbol{\mu}_{i}$ and covariance $\boldsymbol{\Sigma}_{i}$ (Eq. 16-17)

(c5) Regenerate $\mathrm{K}$ weighted particals, and deassign weights(Eq. 18)

(c6) if reached the max iterative times, end; otherwise, return to $(\mathrm{c} 1)$.

D for all $i \in \mathcal{N}$

Calculate self position based on MMSE

or multi-mode, Gaussian distribution is adopted as the worst case. Therefore, the multivariate Gaussian distribution is used to represent the belief between underwater nodes with mean value $\boldsymbol{\mu}_{i}$ and covariance $\Sigma_{i}$ as follows:

$$
\begin{aligned}
\boldsymbol{\mu}_{i} & =\sum_{n=1}^{K} W_{i}[n] \mathbf{x}_{i}[n] \\
\boldsymbol{\Sigma}_{i} & =\frac{\sum_{n=1}^{K} W_{i}[n]\left(\mathbf{x}_{i}[n]-\boldsymbol{\mu}_{i}\right)\left(\mathbf{x}_{i}[n]-\boldsymbol{\mu}_{i}\right)^{T}}{1-\sum_{n=1}^{K}\left(W_{i}[n]\right)^{2}}
\end{aligned}
$$

Regenerate K particles according to Gaussian Approximation, the weight corresponding to each particle is expressed as follows:

$W_{i}[n] \propto \exp \left[-\frac{1}{2}\left(\mathbf{x}_{i}[n]-\boldsymbol{\mu}_{i}\right)^{T} \Sigma_{i}^{-1}\left(\mathbf{x}_{i}[n]-\boldsymbol{\mu}_{i}\right)\right]$

The algorithmic representation of the proposed weighted particles based distributed underwater co-positioning method, WP-DUCP is given in Table 1.

\section{Simulation and Analysis}

To analysis the performance of WP-DUCP algorithm, we considered a $100 m * 100 m$ two-dimensional under- water scenario, in which the number of underwater anchor nodes with high positioning accuracy is $M=13$ and the number of underwater nodes to be located is $N=100$. Anchor nodes are underwater nodes with known position coordinates with high positioning accuracy, and the underwater nodes to be located are randomly distributed in the interested area. The communication distance between any two underwater nodes is equal to the ranging distance, and is set as R. Suppose the ranging noise between any two underwater nodes follows zero mean Gaussian distribution with variance of $\sigma_{i->j}^{2}$. The prior probability density function of the position of the undetermined underwater node $i$ can be denoted as follows:

$$
f\left(\mathbf{x}_{i}\right)=N\left(\mathbf{x}_{i}, \boldsymbol{\mu}_{i}^{(0)}, \boldsymbol{\Sigma}_{i}^{(0)}\right)
$$

On the assumption of independence between the two axes, $\Sigma_{i}^{(0)}=\operatorname{diag}\left(\left[\left(\sigma_{x_{i}}^{(0)}\right)^{2},\left(\sigma_{y_{i}}^{(0)}\right)^{2}\right]\right)$. Set the maximum iteration to be $L=20$. The number of particles in local information representation to be $k=500$. The simulation is to evaluate the performance on terms of convergence, error and computation complexity.

\subsection{Convergence}

In the simulation, consider the standard deviation of ranging error between underwater nodes is set as $/$ sigma $_{i->j}=$ $1 m$, and the standard deviation of the underwater node position is $\sigma_{x_{i}}=\sigma_{y_{i}}=10 \mathrm{~m}$. Compare the proposed WP-DUCP to SDP algorithm[10] and Taylor-DP algorithm[12] in term of convergence speed. The comparison results of positioning performance are shown in figure 2 .

It can be seen from Fig 2 that, the positioning error is decreased to convergence with the iteration. The SDP algorithm tends to convergence after 8 iterations, and RMSE error can reach about $1.4 \mathrm{~m}$. The TaylorDP method needs about 10 times, and the RMSE error is more than $1.6 \mathrm{~m}$. The proposed WP-DUCP method shows superiority with about 5-6 iterations, and the convergence error of RMSE can reach about $1.2 \mathrm{~m}$. It attributes to the adoption of weighted particles to represent and transmit messages. The representation of a large number of particles makes it very close to the real distribution of information, and fast convergence can be achieved.

\subsection{Positioning Accuracy Analysis}

Positioning accuracy is the most important support point of underwater cooperative navigation. In this part, the 


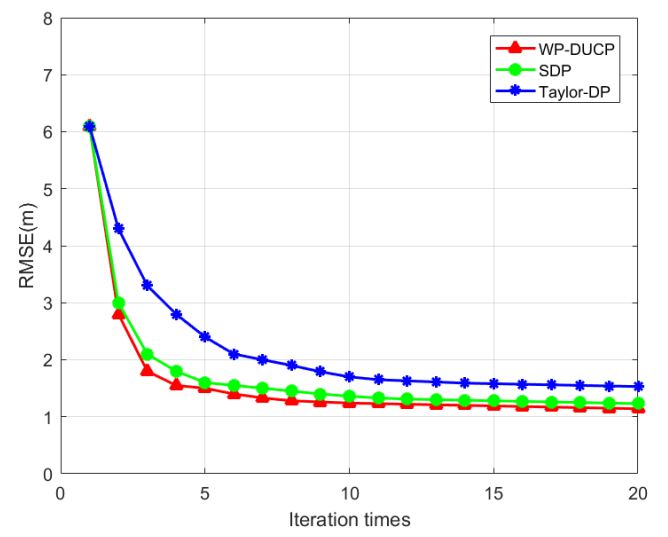

Fig. 2 Convergence Comparison Chart

positioning accuracy is simulated under the same simulation as in section 4.1, and the results are shown in Fig 3.

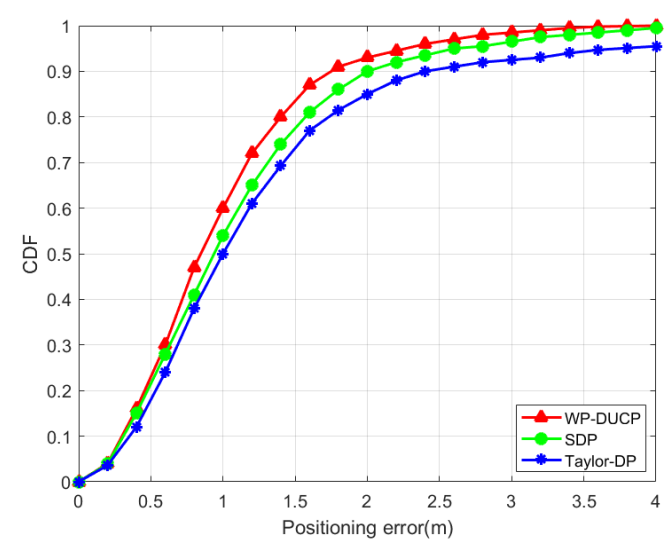

Fig. 3 Convergence Comparison Chart

As can be seen from Fig 3, the positioning performance of Taylor-DP algorithm is relatively poor. When the positioning error is less than $3 \mathrm{~m}$, CDF can only reach $91 \%$, indicating that nearly $10 \%$ of the positioning accuracy of cooperative nodes has not been substantially improved. SDP method is better than Taylor-DP algorithm. When the positioning error is less than $3 \mathrm{~m}$, CDF can reach $96 \%$, indicating that the positioning accuracy of most cooperative nodes has been improved. When the positioning error is less than $3 \mathrm{~m}$, the WPDUCP algorithm proposed in this paper can guarantee that the positioning performance of $98 \%$ of cooperative nodes is improved. This is attribute to the utilization of the summation and product theory of factor graphs to convert the global optimum into the local optimum product, and it can effectively improve the positioning accuracy of underwater collaborative nodes by spread-
Table 2 Complexity and communication burden comparison

\begin{tabular}{lll}
\hline Algorithm & Computation Complexity & Communication Burden \\
\hline SDP & $O\left(K^{2} N_{i}\right)$ & K parameters \\
& & and K partical weights \\
Taylor-DP & $O\left(K N_{i}\right)$ & K parameters \\
WP-DUCP & $O\left(N_{i}\right)$ & K parameters \\
\hline
\end{tabular}

ing through all the cooperative positioning nodes with confidence.

\subsection{Complexity Analysis}

Computational complexity and communication cost are the decisive factors for the real-time performance of the underwater positiong algorithm. In this paper, Denote the number of particle parameters as $K$ and the number of neighbor nodes of the undetermined node to be localized as $N_{i}$. The complexity comparison are shown in table 4.3 .

In terms of the computation complexity, the SDP algorithms failed in the comparison, as its complexity is proportion to the square of $K$ and $N_{i}$. The computation complexity of Taylor-DP algorithm is proportion to the product of $K$ and $N_{i}$, while that of the proposed WP-DUCP is proportion to $N_{i}$. The communication burden of SDP algorithm is higher than that of the other two algorithms. $K$ parameters and $K$ weight coefficients need to be transferred at per node in SDP algorithm, while only $K$ parameters are needed at per node in Taylor-DP and WP-DUCP algorithm.

\subsection{Iteration Analysis}

In this simulation, set the anchor nodes at the predefined position, and distribute undertermined nodes randomly in the considered $100 \mathrm{~m}^{*} 100 \mathrm{~m}$ two-dimensional underwater scenario, as shown in Fig. 4. In this figure, the red asterisks represent the anchor nodes, while the green circles denotes the undetermined nodes. The localization results after 10 iterations is shown as the red asterisks. As can be seen from this figure, the localization performance in the centre is better than that in the corner, since more anchor nodes are included.

The colour temperature of the positioning error of the 100 undetermined nodes within each iteration is shown in Fig. 5. Most positioning error is below $1.4 \mathrm{~m}$, while the worst error is nearly $12 \mathrm{~m}$.

\section{Experiment and Analysis}

An anechoic tank test is conducted to verify the performance of the WP-DUCP localization method. The 


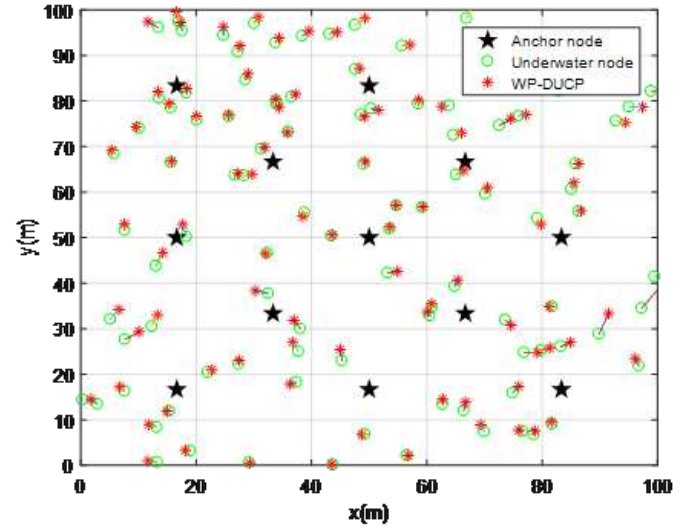

Fig. 4 WP-DUCP localization results

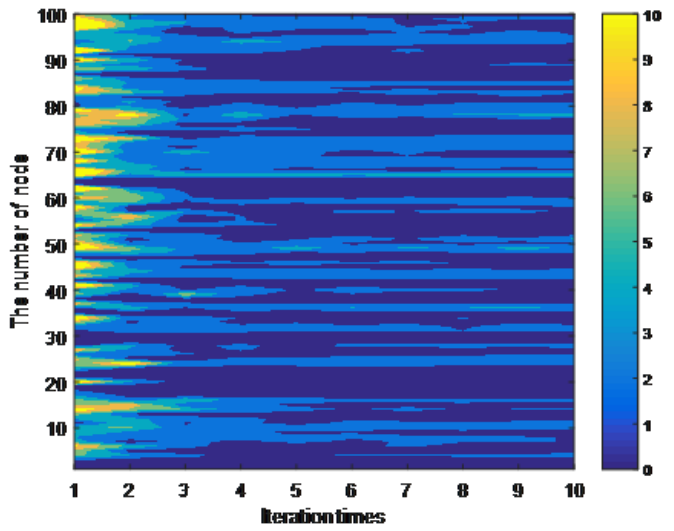

Fig. 5 WP-DUCP localization error

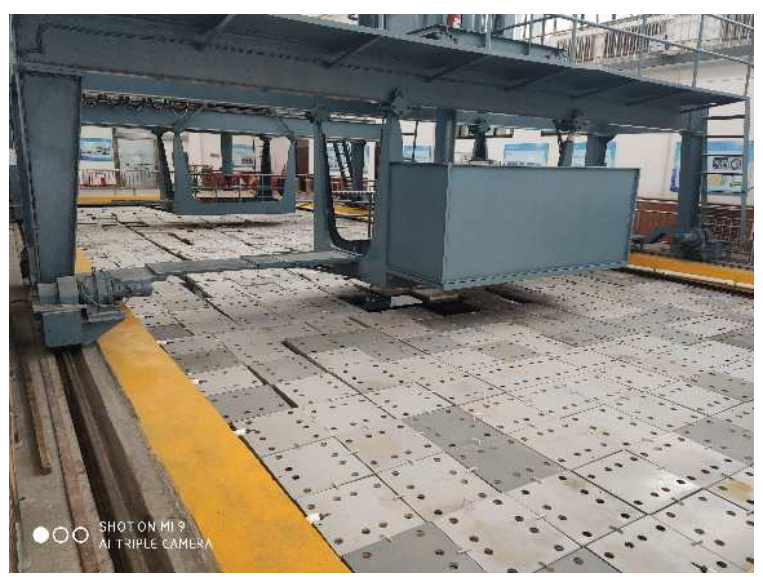

Fig. 6 The anechoic tank environment

tank is covered with silencing structure to avoid echo, as shown in Fig 6 . The self-designed underwater modem as shown in Fig 7 is adopted as the communication node. It is configured to work in ranging mode, and the precision is within 0.1 meter. The experiment is conducted in three scenarios, each lasts 75 minutes and collects 75,000 sets of data.

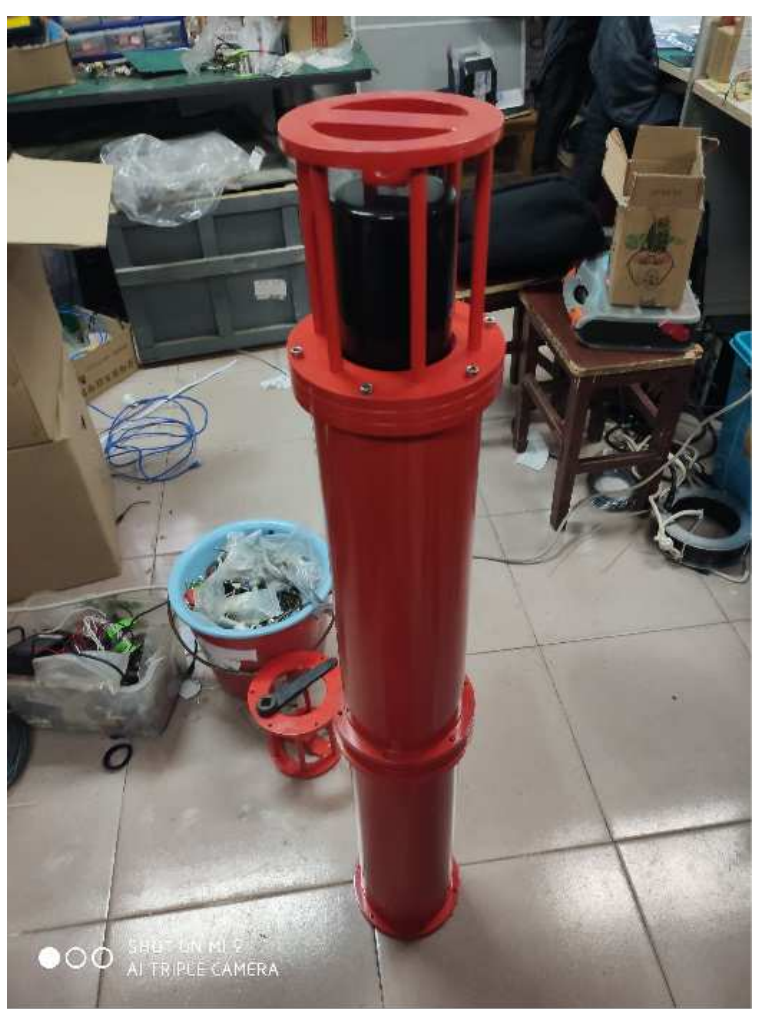

Fig. 7 The adopted communication node

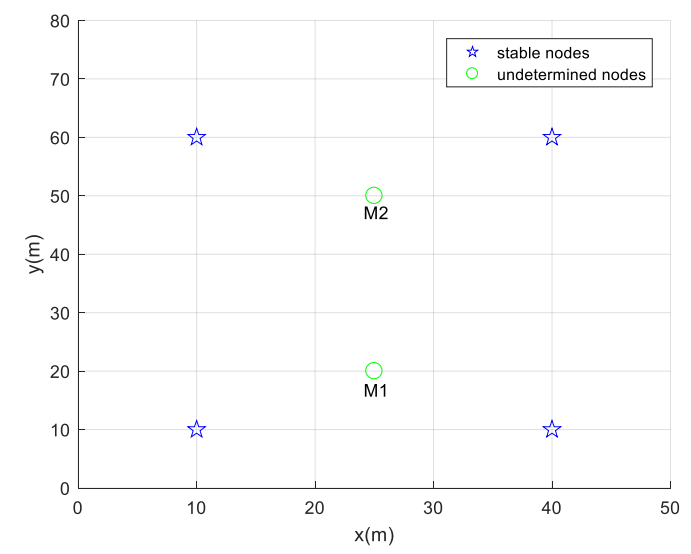

Fig. 8 Nodes Geometry in Scenario 1

\subsection{Scenario 1}

In this scenario, four anchor nodes are placed at the four corner of a $3 m * 5 m$ square, and the two undetermined nodes are placed on the axis within the square, as shown in Fig 8. The initial position estimation error is within 1 meter, while the ranging error is within 0.1 meter.

Adopted the proposed co-location method based on the collected ranging measurements, the obtained position estimation is shown in Fig 9(a), while the RMSE of the position estimation of each undetermined node is shown in Fig 9(b). The CDF of the whole network is shown in Fig 10. 


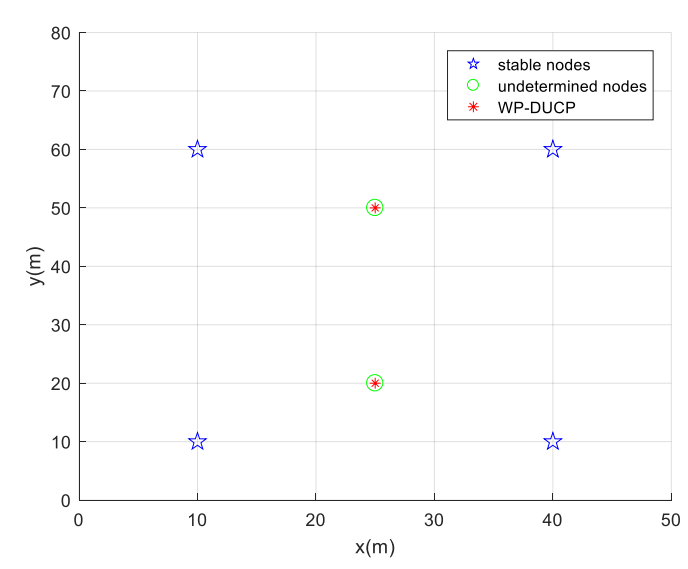

(a) Position Estimation Result

Fig. 9 Co-location results of Scenario 1

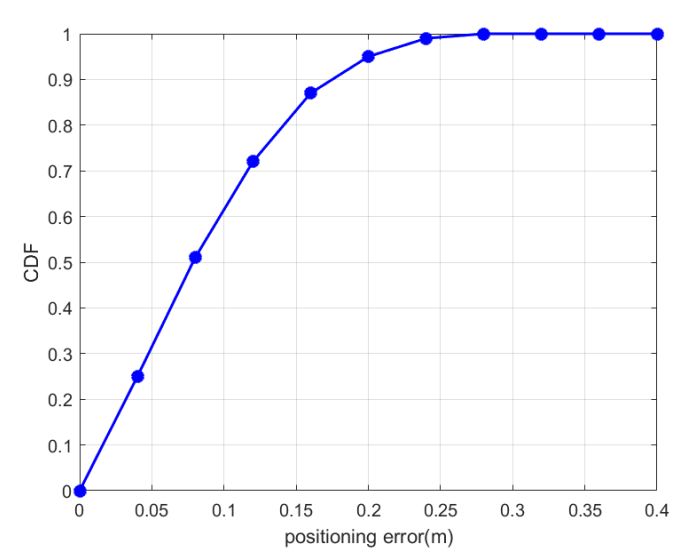

Fig. 10 Positioning performance of WP-DUCP algorithm of Scenario 1

As seen from Fig 9, the position estimation error is approaching the ranging precision, that is to say, the method could alleviate the effect of the initial position ambiguity. This is attributes to the belief propagation within the factor graph. The positioning error of M1 and M2 are almost equal due to the symmetry geometry setting. As can be seen from figure 10, about $95 \%$ of the collected data, the collaborative positioning accuracy of the whole network in scenario 1 can be achieved within 0.2 meter after multiple iterations. The experiment results show that, the WP-DUCP algorithm has low computational complexity and communication overhead. It is attribute to the local information exchange in the for$\mathrm{m}$ of parameters, and the results is consistent with the theoretical analysis. It is predicted to have a better positioning performance when the underwater nodes are in a stable state.

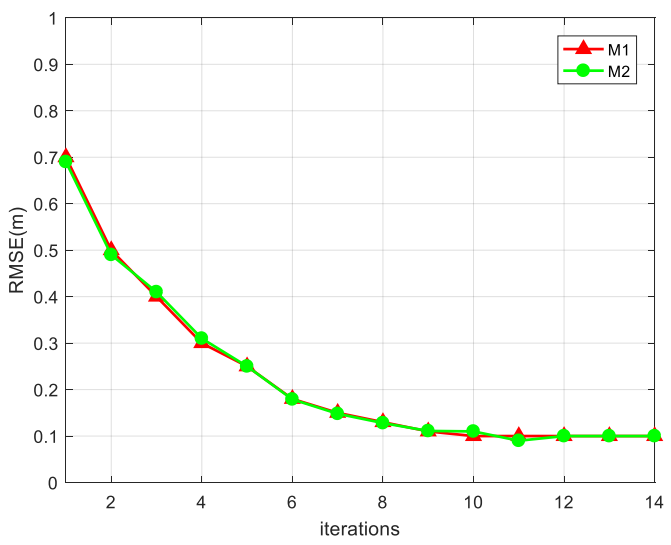

(b) RMSE vs Iteration

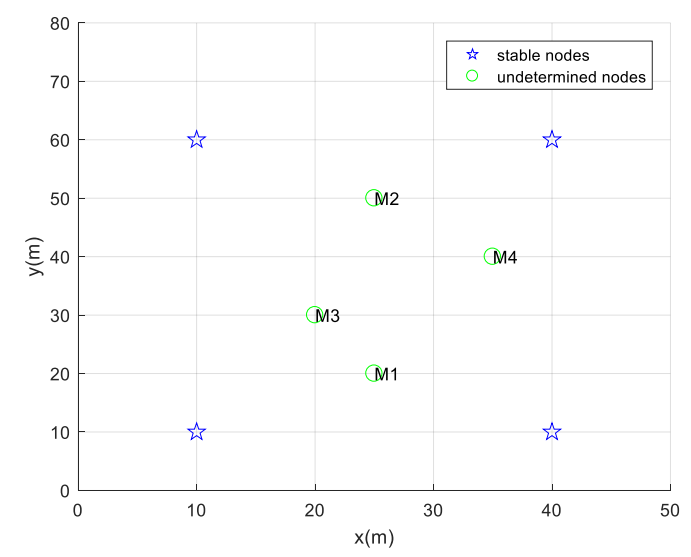

Fig. 11 Scenario 2

\subsection{Scenario 2}

In scenario 2, to test the performance of the proposed method to nodes not on the axis, we add another two undetermined nodes off the axis on basis of the network setting in scenario 1, as shown in Fig 11.

Adopted the proposed co-location method based on the collected ranging measurements, the obtained position estimation is shown in Fig 12(a), while the RMSE of the position estimation of each undetermined node is shown in Fig 12(b). The CDF of the whole network is shown in Fig 13.

As can be seen from figure 12, the positioning accuracy of the four nodes M1, M2, M3 and M4 after convergence reach about $0.1 \mathrm{~m}$, similar to that of scenario 1. It shows that the WP-DUCP algorithm can also realize the fast convergence when the number of nodes increases. This is because the WP-DUCP algorithm takes part in the positioning solution by selecting the nodes with high confidence in the leading node, and eliminates the influence of the nodes with poor positioning accura- 


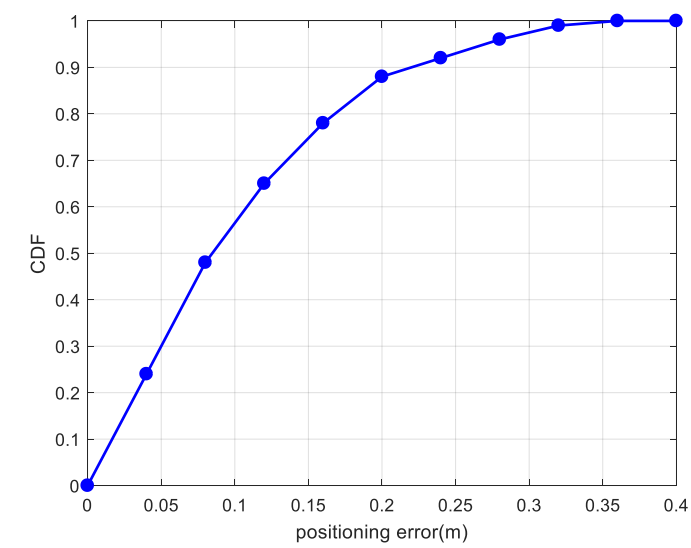

Fig. 13 Positioning performance of WP-DUCP algorithm of Scenario 2

cy. In the 75,000 sets of test data, approximately $90 \%$ of the positioning accuracy can be achieved, as shown in figure 13. The experimental results verified that the WP-DUCP algorithm is consistent with the theoretical analysis.

\subsection{Scenario 3}

In underwater network, it is difficult for anchor nodes to sustain in periphery all the time. Sometimes, due to the mobile characteristics of ocean waves, undetermined nodes may appear outside the square. On the basis of network setting of scenario 2, we move one undetermined node outside the square, as shown in figure 14, to further verify the performance of WP-DUCP method.

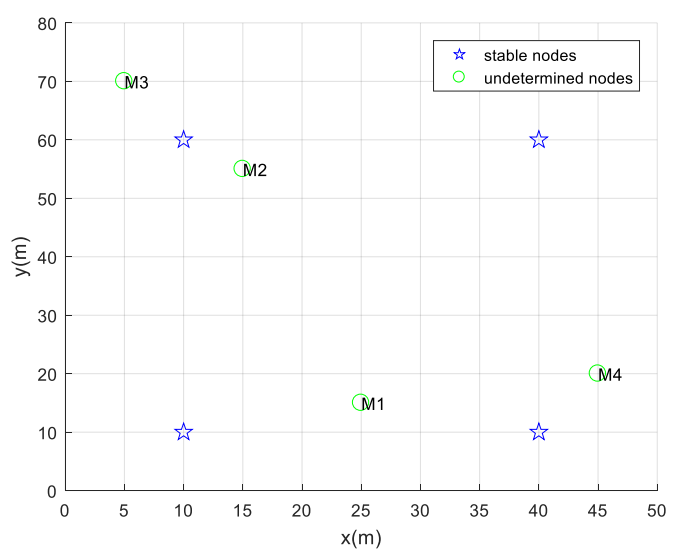

Fig. 14 Scenario 2

Adopted the proposed co-location method based on the collected ranging measurements, the obtained position estimation is shown in Fig 15(a), while the RMSE of the position estimation of each undetermined node is shown in Fig 15(b). The CDF of the whole network is shown in Fig 16.

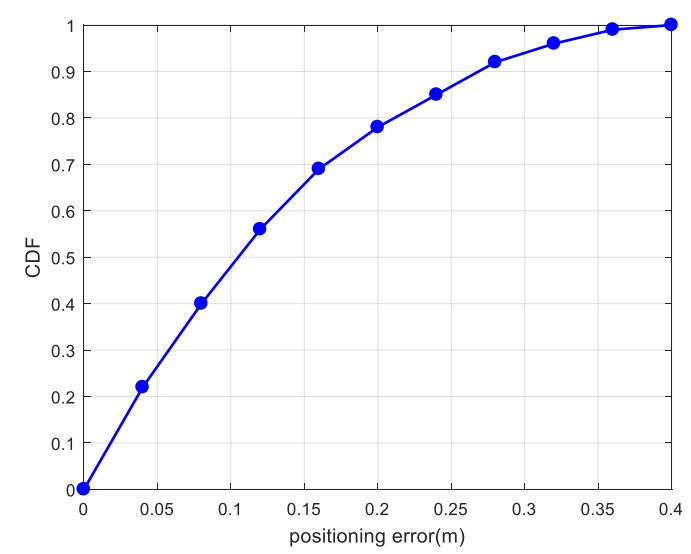

Fig. 16 Positioning performance of WP-DUCP algorithm of Scenario 3

As can be seen from figure 16, the positioning accuracy of the two inner nodes $\mathrm{M} 1, \mathrm{M} 2$ reach about $0.1 \mathrm{~m}$, while that of the two outer nodes M3 and M4 after convergence reach about $0.12 \mathrm{~m}$. It shows that the WPDUCP algorithm can also realize the fast convergence with a slight worse performance for outer nodes. This is contribute to the data fusion conducted in the belief propagation of the WP-DUCP algorithm with high confidence in the leading node. In the 75,000 sets of test data, approximately $90 \%$ of the positioning accuracy can be achieved to $0.28 \mathrm{~m}$, as shown in figure 16 . The experimental results verified that the WP-DUCP algorithm is consistent with the theoretical analysis.

\section{Conclusion}

Co-localization method transplanting from wireless research field suffers the problem of difficult communication and complex channel fading when adopted in underwater scenarios. Taking these factors into account, we proposed a factor graph weighted particles distributed underwater co-positioning (WP-DUCP) approach. It is verified by simulation from three aspects: convergence speed, positioning error and computational complexity. The simulation results shows that the proposed WPDUCP method reach convergence after 9 iterations, and its convergence speed is superior to other co-location method. The positioning accuracy is increased by more than $20 \%$, compared with other collaborative localization algorithm. The complexity of algorithm also has a certain improvement. The experiment was carried out in the anechoic tank. It shows that under the condition of three chosen distribution of underwater nodes, 
the positioning accuracy of underwater nodes can reach $0.1 \mathrm{~m}-0.3 \mathrm{~m}$, which is promising to multiple application in underwater joint positioning network.

Acknowledgements The authors would like to thank editors for rigorous work and the anonymous reviewers for their comments and suggestions. This work was supported in part by the National Natural Science Foundation of China under Grant 61801394 and 61803310, in part by Fundamental Research Funds for the Central Universities under Grant 3102019HHZY030013 and Grant G2019KY05206, and in part by the Natural Science Basic Research Plan in Shaanxi Province of China under Grant 2020JQ-202, and in part by China Postdoctoral Science Foundation under Grant 2020M673482 and $2020 \mathrm{M} 673485$

\section{Conflict of interest}

On behalf of all authors, the corresponding author states that there is no conflict of interest.

\section{References}

1. Shao J, Luo D, Xu Y, et al. Cooperative Path Planning for Multiple Robots With Motion Constraints in ObstacleStrewn Environment[J]. IEEE ACCESS, 2019,132286132301.

2. Picardi G, Chellapurath M, Lacoponi S, et al. Bioinspired underwater legged robot for seabed exploration with low environmental disturbance[J].SCIENCE ROBOTICS, 2020, 42(5):1-16

3. Zhang Dangquan, Ashraf Muhammad Aqeel, Liu Zhenling, et al. Dynamic modeling and adaptive controlling in GPS-intelligent buoy (GIB) systems based on neural-fuzzy networks[J].AD HOC NETWORKS, 2020, 103(1):34-46

4. Chengkai Tang, LinglingZhang, Yi Zhang, et al. Factor Graphs-assisted Distributed Cooperative Positioning Algorithm in GNSS system[J]. SENSORS, 2018,18(11):1-12

5. Fernandez-Bes J, Azpicueta-Ruiz L A, et al. Distributed estimation in diffusion networks using affine least-squares combiners [J]. Digital Signal Processing, 2015, 36(1):1-14.

6. Mohsen Rohani, Denis Gingras, Dominique G. A Novel Approach for Improved Vehicular Positioning Using Cooperative Map Matching and Dynamic Base Station DGPS Concept[J]. IEEE Transactions on Intelligent Transportation Systems, 2016, 17(1):1-40.

7. Zhang Lingling, Tang Chengkai, Chen, Peilin, et al.Gaussian Parameterized Information aided Distributed Cooperative Underwater Positioning Algorithm[J].IEEE Access, 2020, 64634-64645.

8. B. akmak, D. N. Urup, F. Meyer, T. Pedersen, B. H. Fleury and F. Hlawatsch, Cooperative Localization for Mobile Networks: A Distributed Belief PropagationMean Field Message Passing Algorithm[J]. IEEE Signal Processing Letters, 2016, 23(6): 828-832.

9. Helmut Hlavacs, Karin Anna Hummel. Cooperative Positioning when Using Local Position Information: Theoretical Framework and Error Analysis[J]. IEEE Transactions on Mobile Computing, 2013, 12(10):1366-1376.

10. Ghari Pouya M, Shahbazian Reza, Ghorashi Seyed Ali. Maximum entropy-based semi-definite programming for wireless sensor network localization[J]. IEEE Internet of Things Journal,2019,6(2):3480-3491.
11. Jianhua Cui, Zhongyong Wang, Chuanzong Zhang, et al. Message Passing localisation algorithm combining BP with VMP for mobile wireless sensor networks[J]. IET Communications, 2017, 11(7):1106-1113.

12. Wang Yue-Jiao, Ma Zhong, Tang Xue-Han. Distributed implementation of iterative Kalman filter localization with taylor expansion for wireless sensor networks[J]. Journal of Computers, 2019,30(3):44-58.

13. Van Nguyen T, Jeong Y, Shin H, et al. Least square cooperative localization[J]. IEEE Transactions on Vehicular Technology, 2015, 64(4):1318-1330.

14. Buehrer R M, Wymeersch H, Vaghefi R M. Collaborative sensor network localization: algorithms and practical issues[J]. Proceedings of the IEEE, 2018, 106(6):1089-1114.

15. Hyowon K, Won C S, Sunwoo K. Connectivity information-aided belief propagation for cooperative localization[J]. IEEE Wireless Communications Letters, 2018, 7(6): 1010-1013.

16. Jouhari Mohammed, IbrahimiKhalil, Tembine Hamidou, et al.Underwater Wireless Sensor Networks: A Survey on Enabling Technologies, Localization Protocols, and Internet of Underwater Things[J]. IEEE Access, 2019, p:96879-96899 17. Gloria Soatti, Monica Nicoli, Nil Garcia, et al. Implicit Cooperative Positioning in Vehicular Networks[J]. IEEE Transactions on Intelligent Transportation Systems, 2018, 19(12): 3020-3033. 


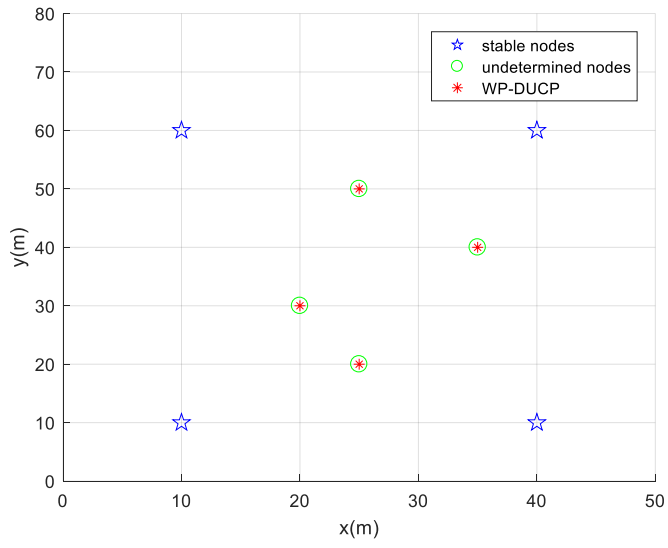

(a) Position Estimation Result

Fig. 12 Co-location results of Scenario 2

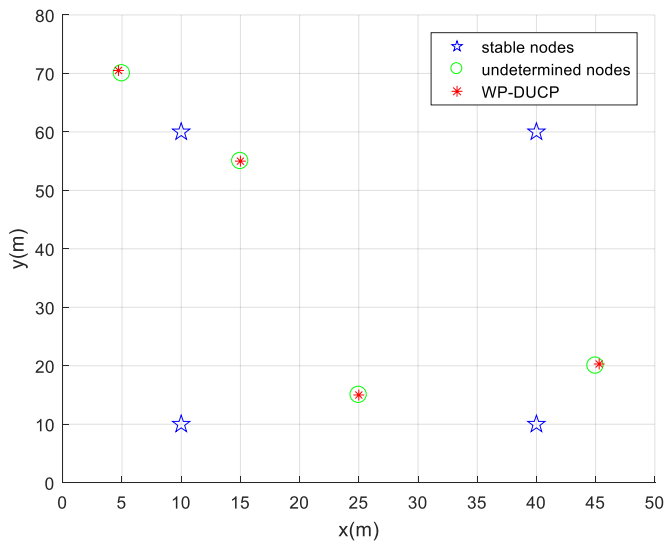

(a) Position Estimation Result

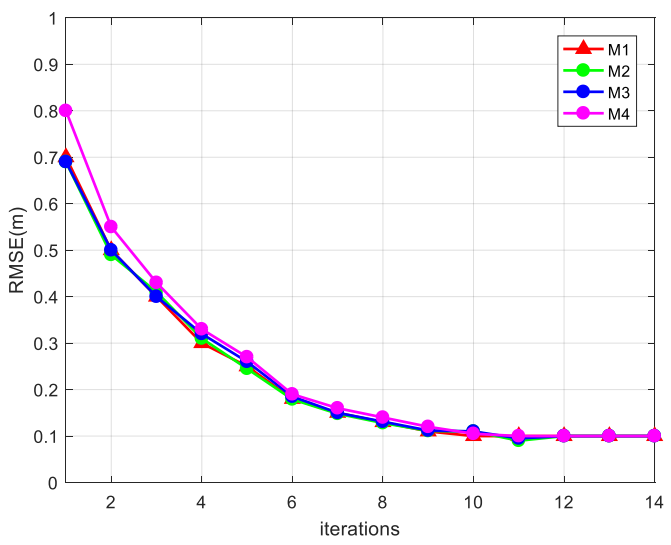

(b) RMSE vs Iteration

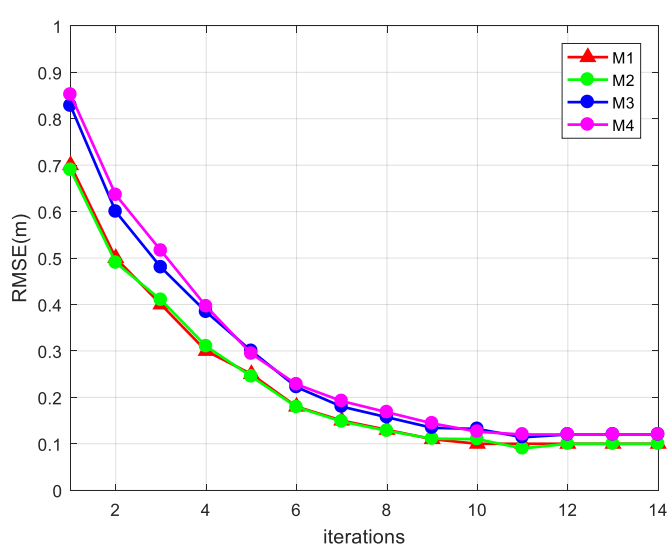

(b) RMSE vs Iteration

Fig. 15 Co-location results of Scenario 2 
Figures

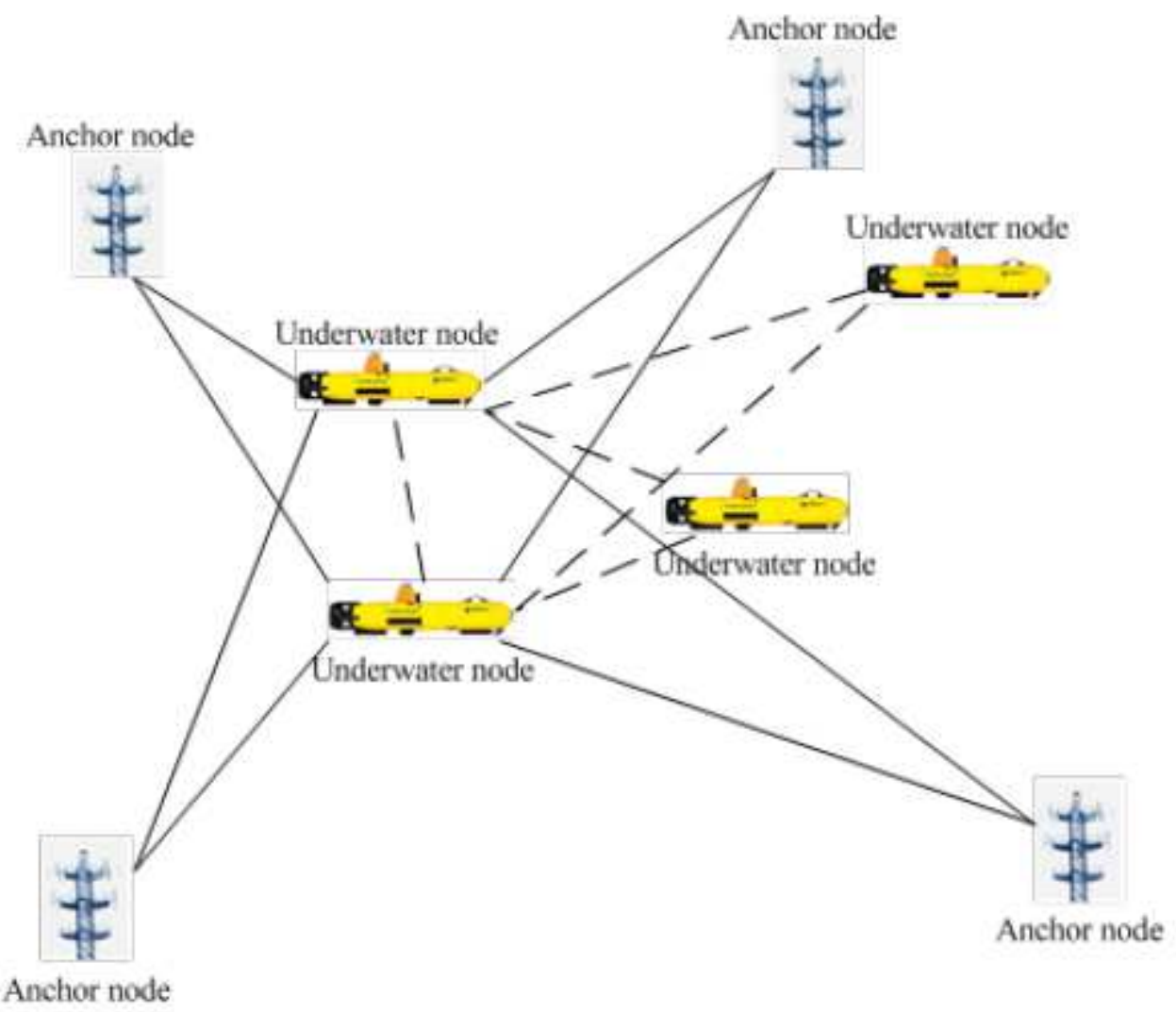

Figure 1

Underwater Co-localization Model

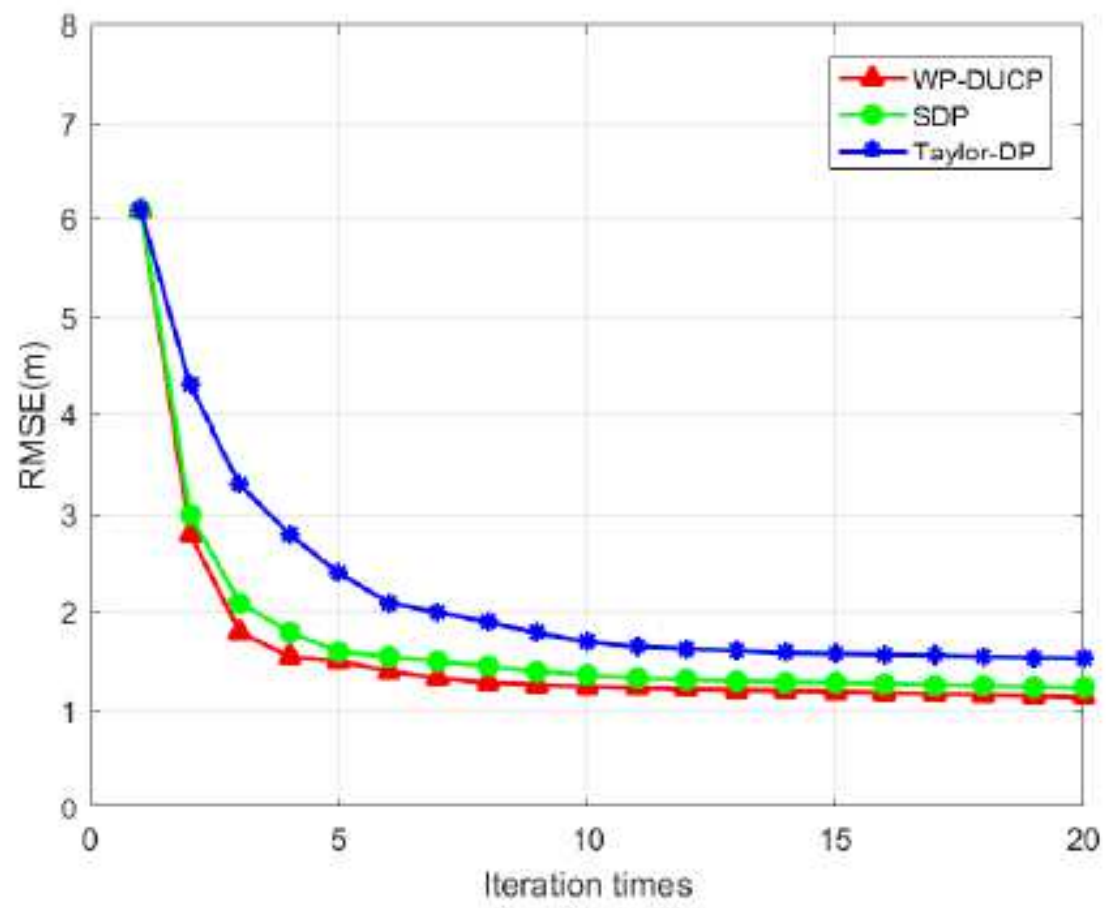


Figure 2

Convergence Comparison Chart

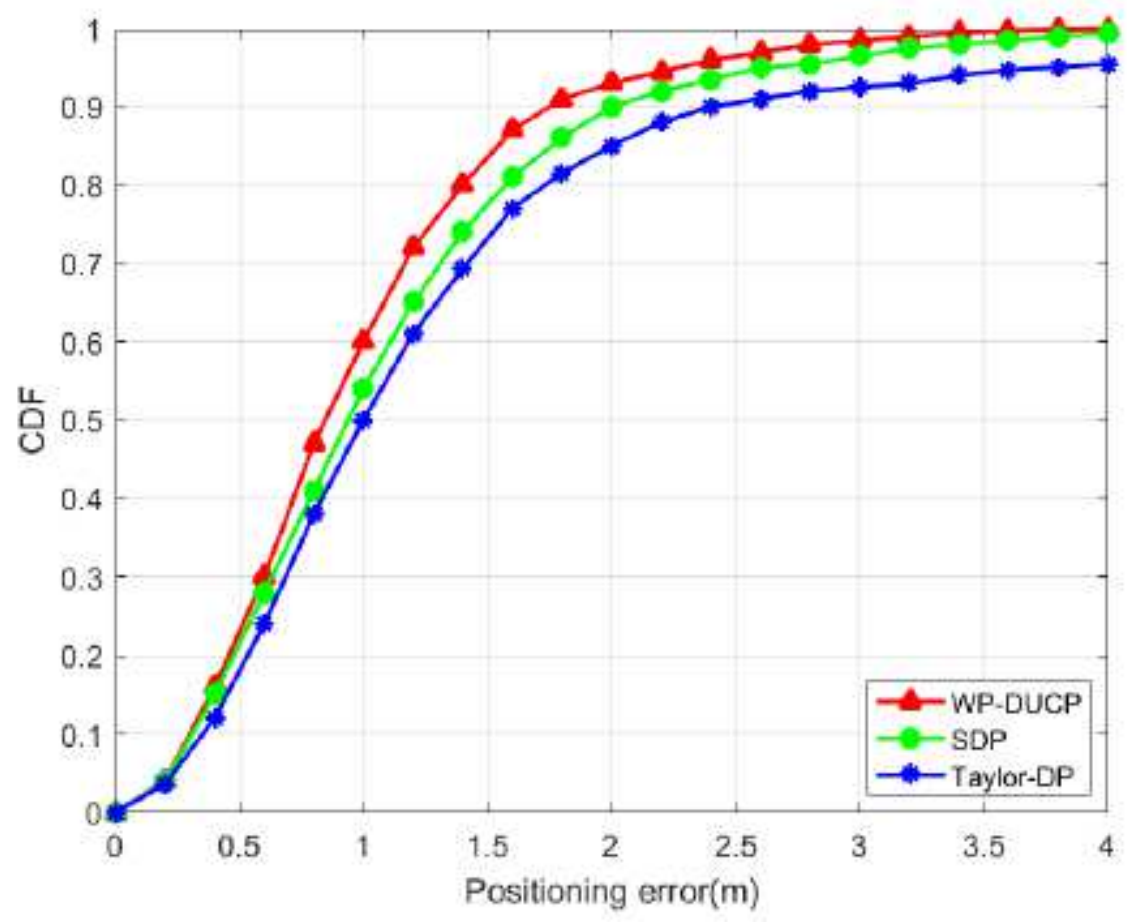

Figure 3

Convergence Comparison Chart

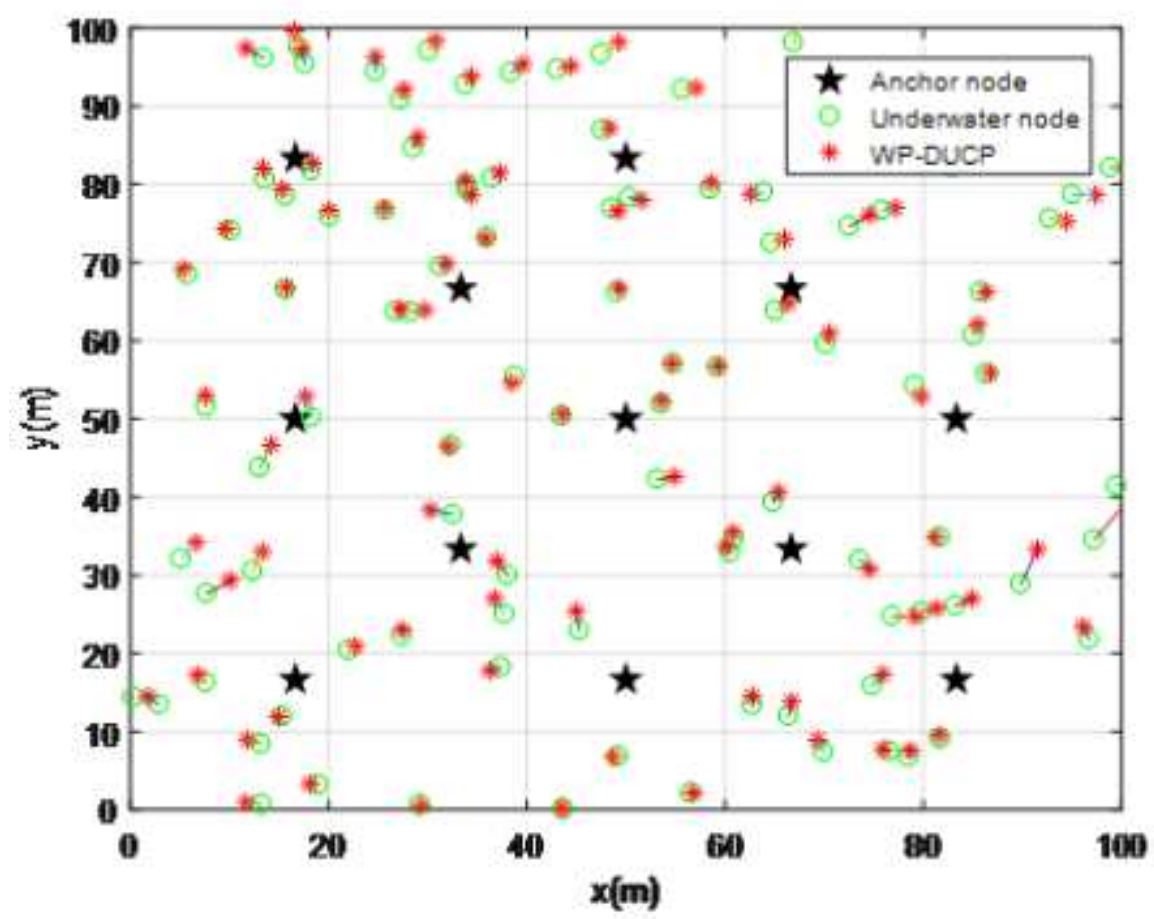

Figure 4

WP-DUCP localization results 


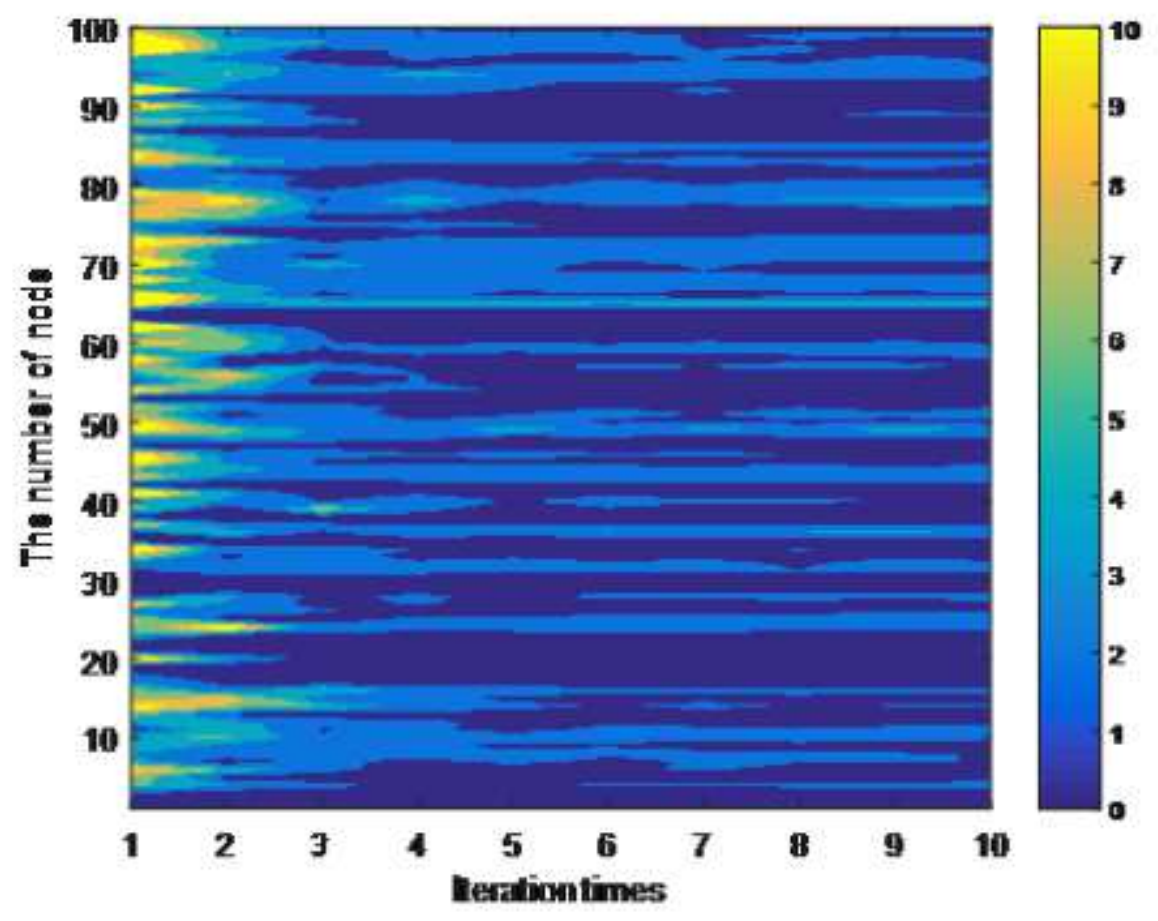

Figure 5

WP-DUCP localization error

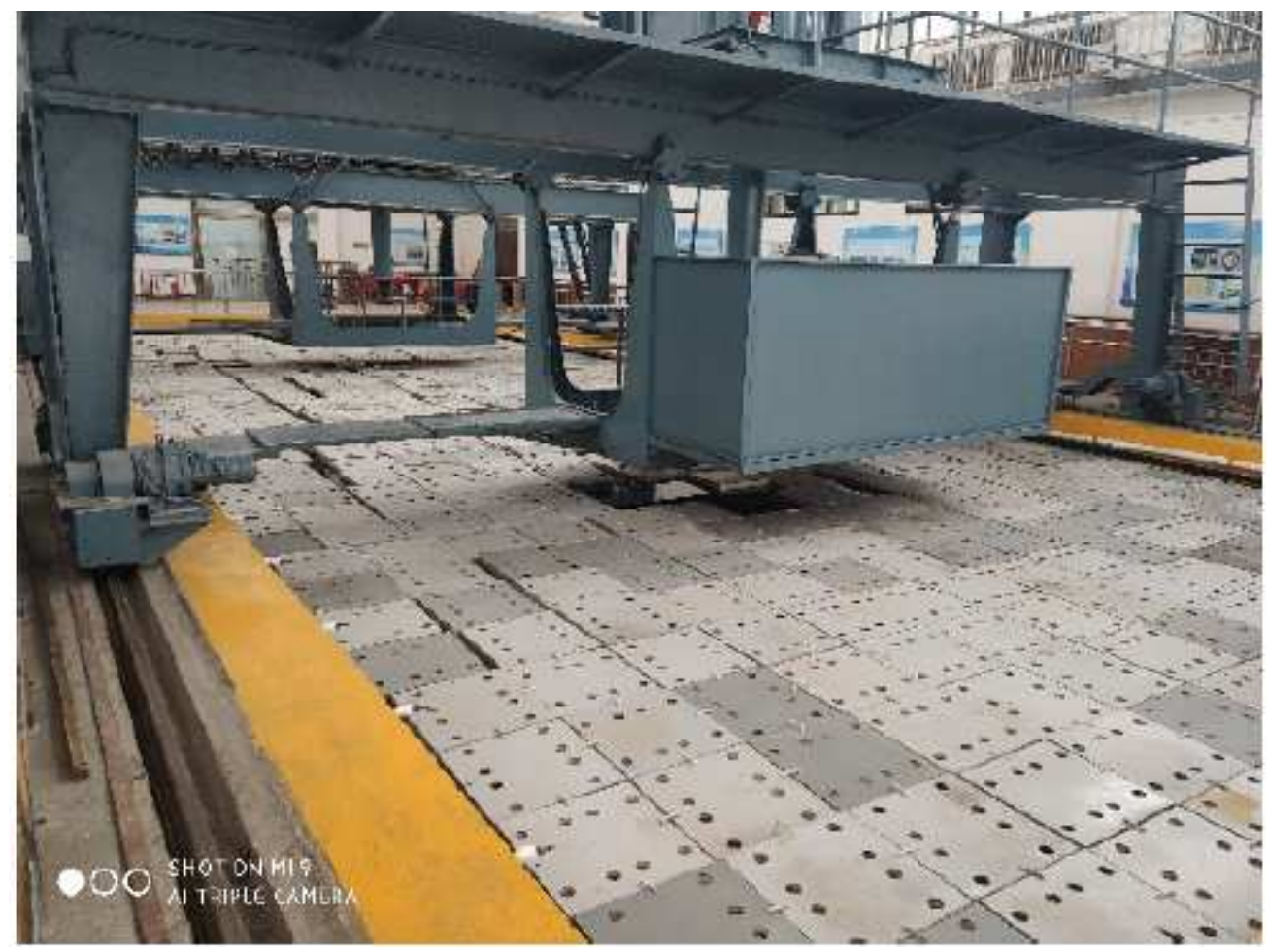

Figure 6

The anechoic tank environment 


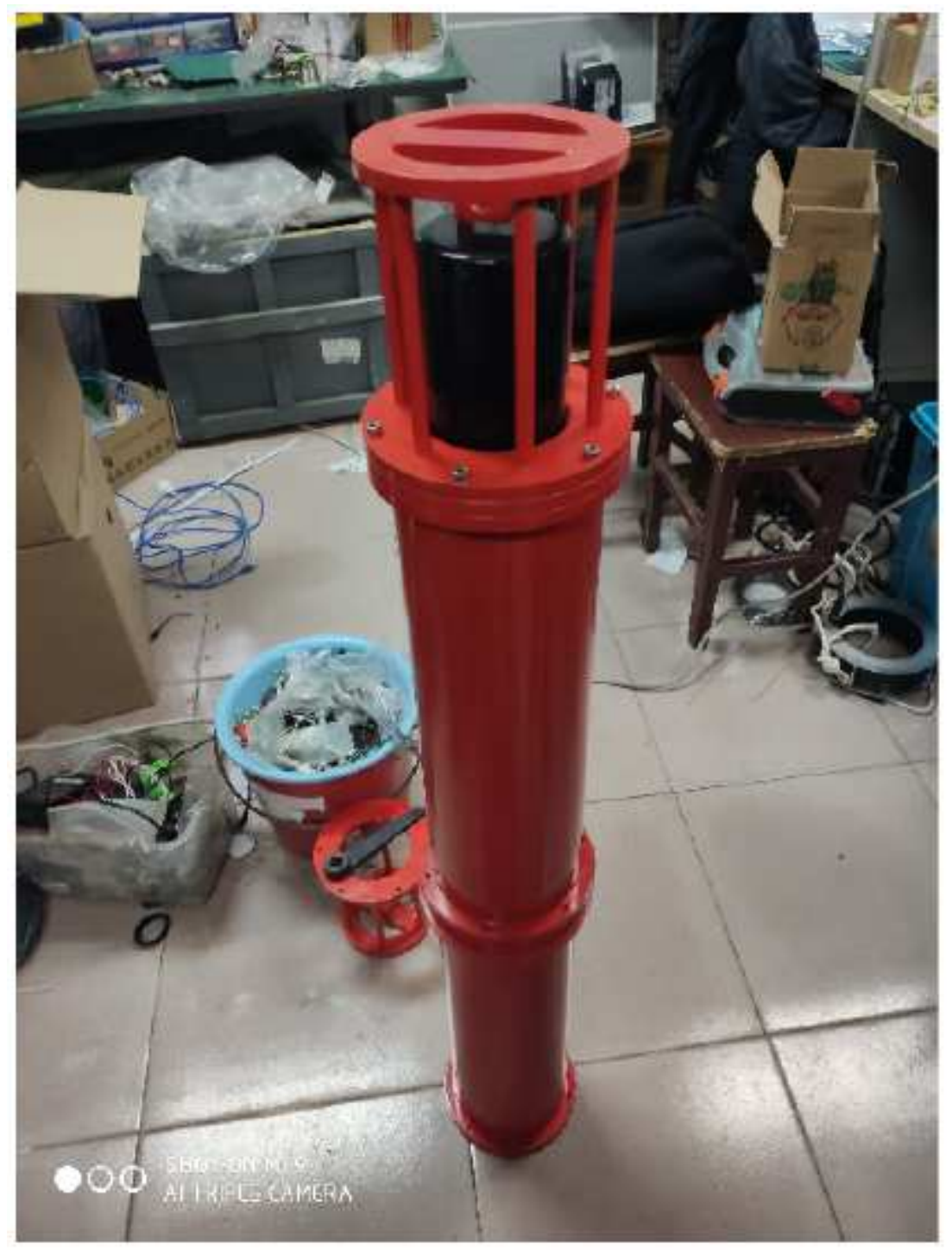

Figure 7

The adopted communication node

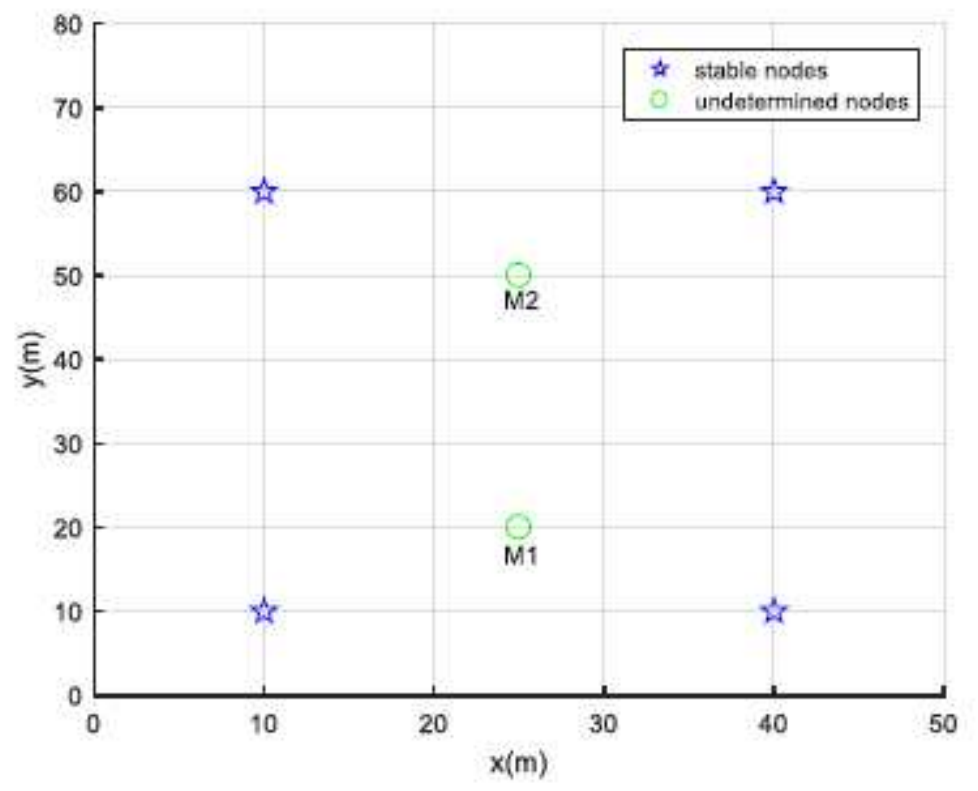


Figure 8

Nodes Geometry in Scenario 1
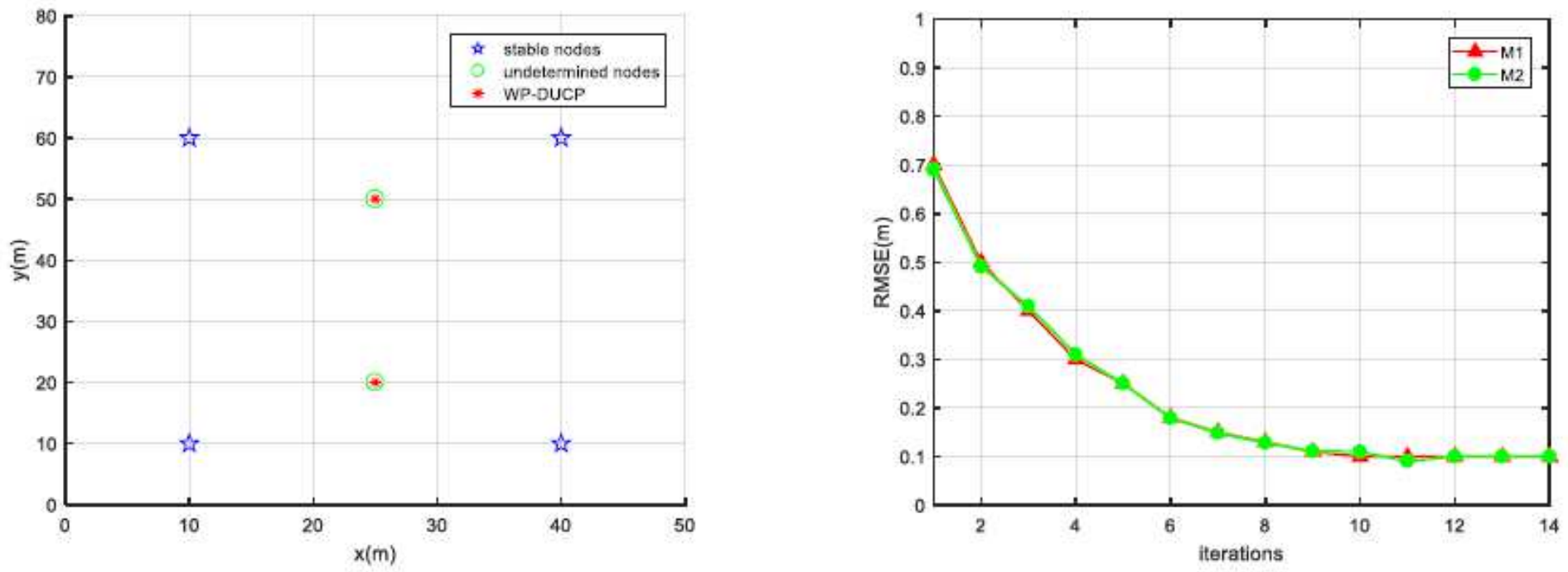

Figure 9

Co-location results of Scenario 1 a) Position Estimation Result (b) RMSE vs Iteration

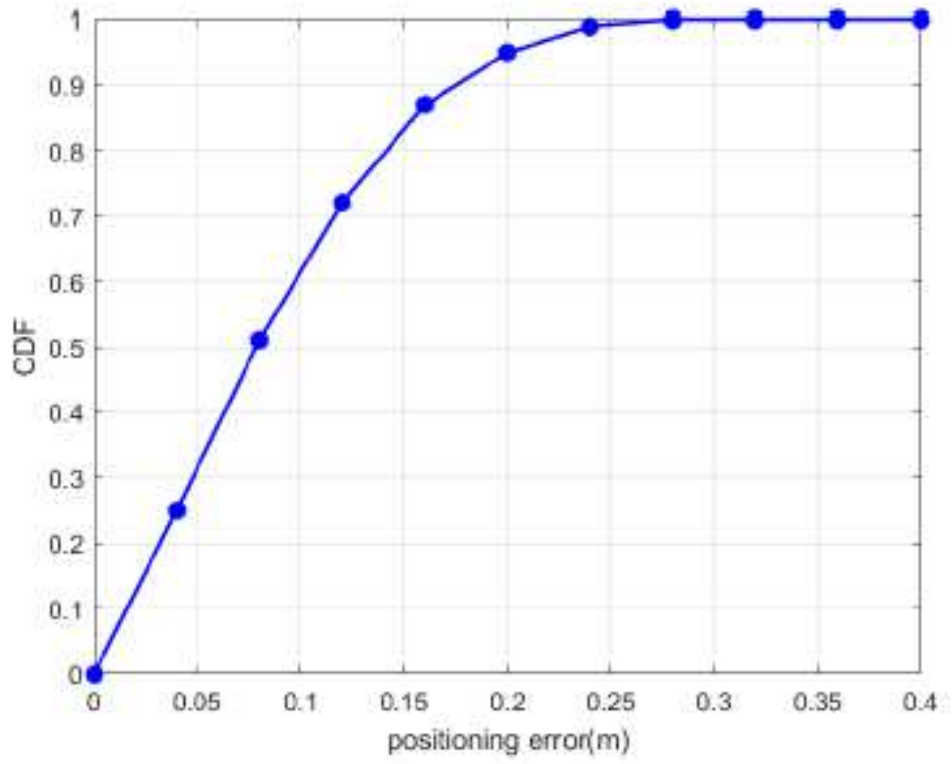

Figure 10

Positioning performance of WP-DUCP algorithm of Scenario 1 


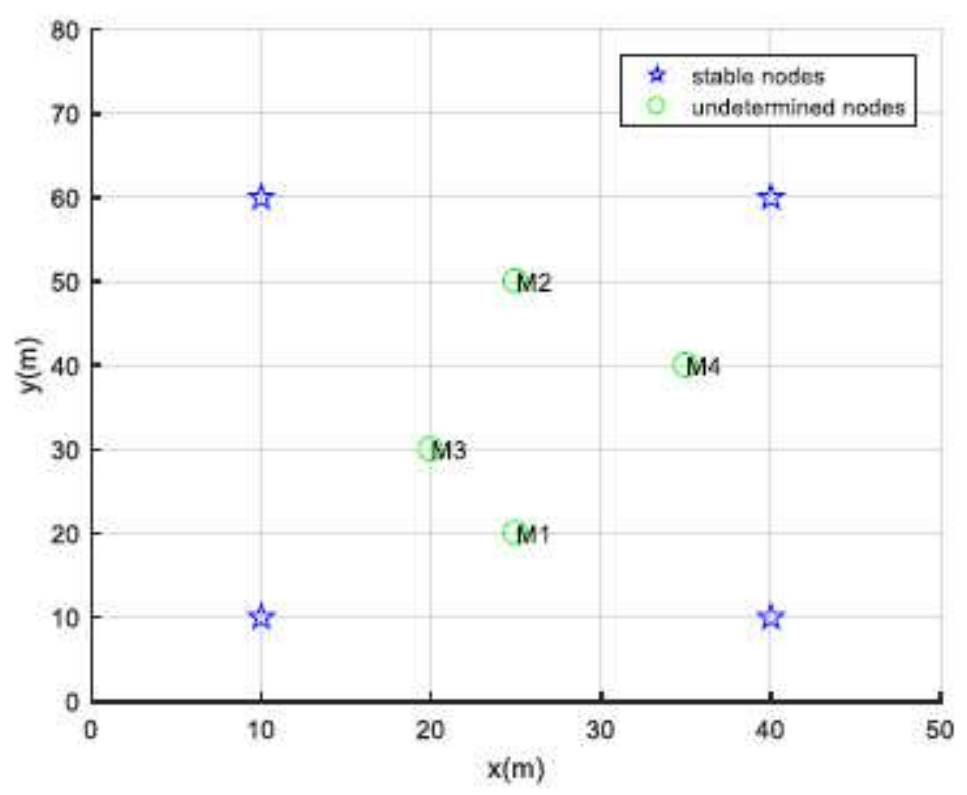

Figure 11

Scenario 2
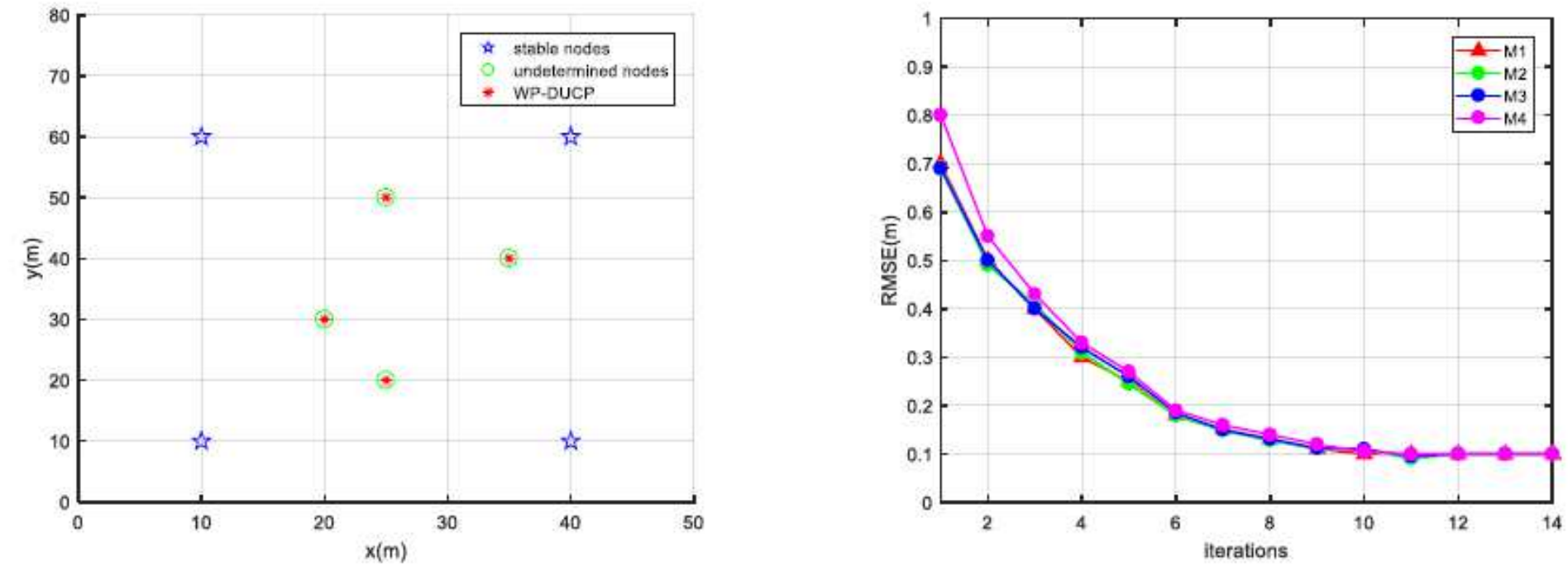

Figure 12

Co-location results of Scenario 2 (a) Position Estimation Result (b) RMSE vs Iteration 


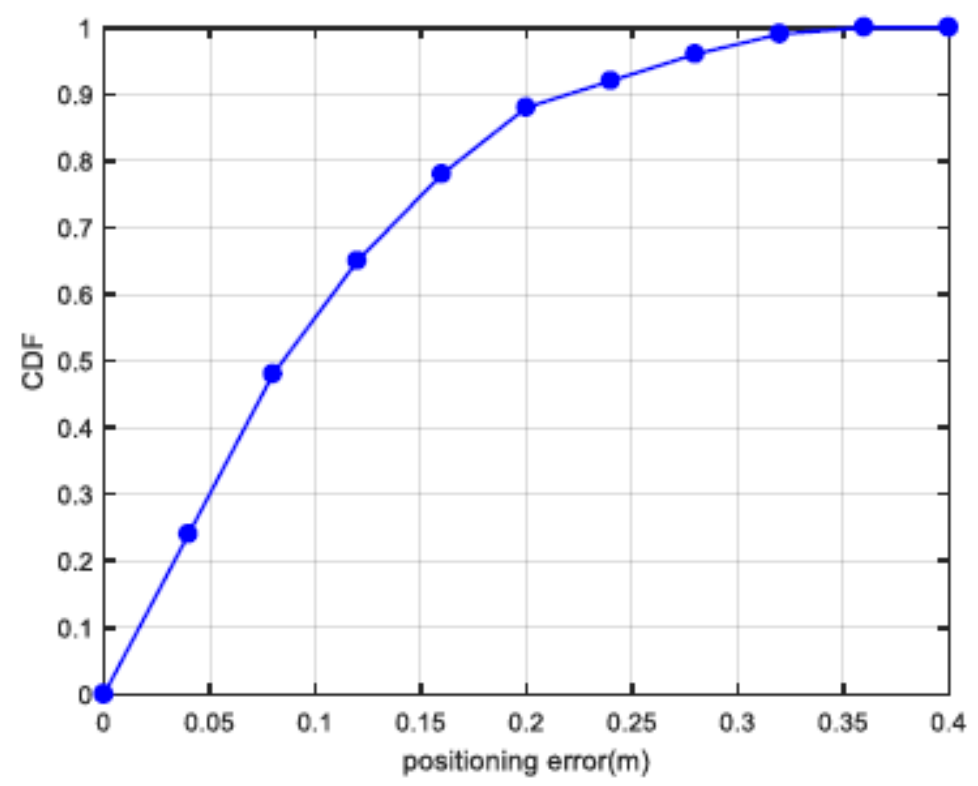

Figure 13

Positioning performance of WP-DUCP algorithm of Scenario 2

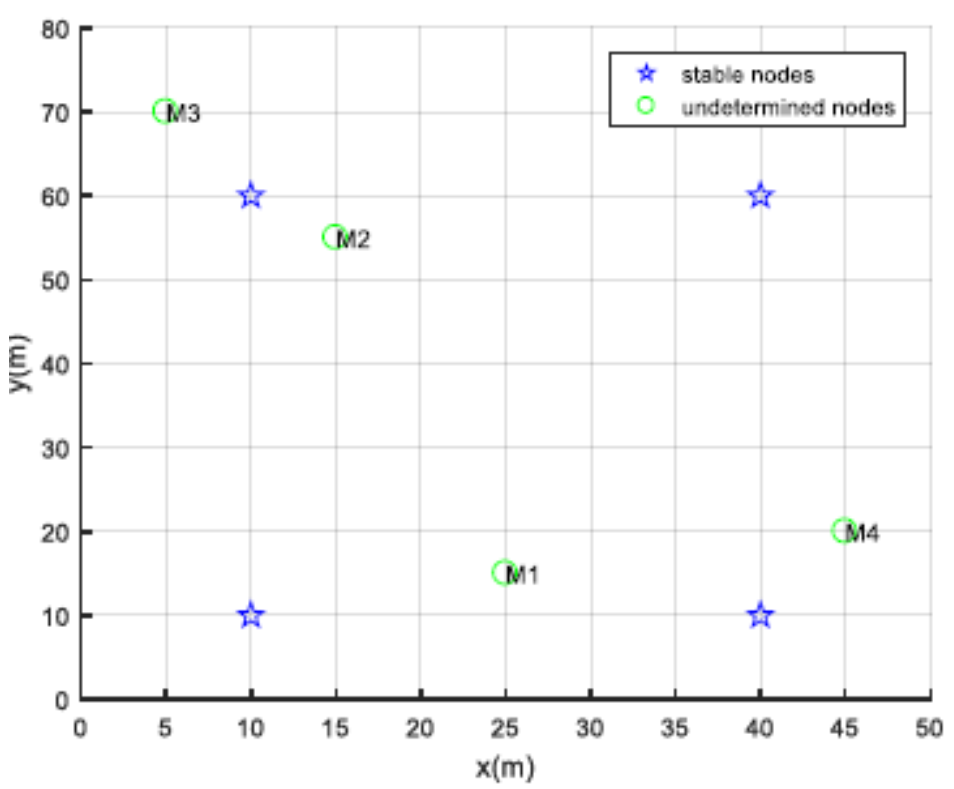

Figure 14

Scenario 2 

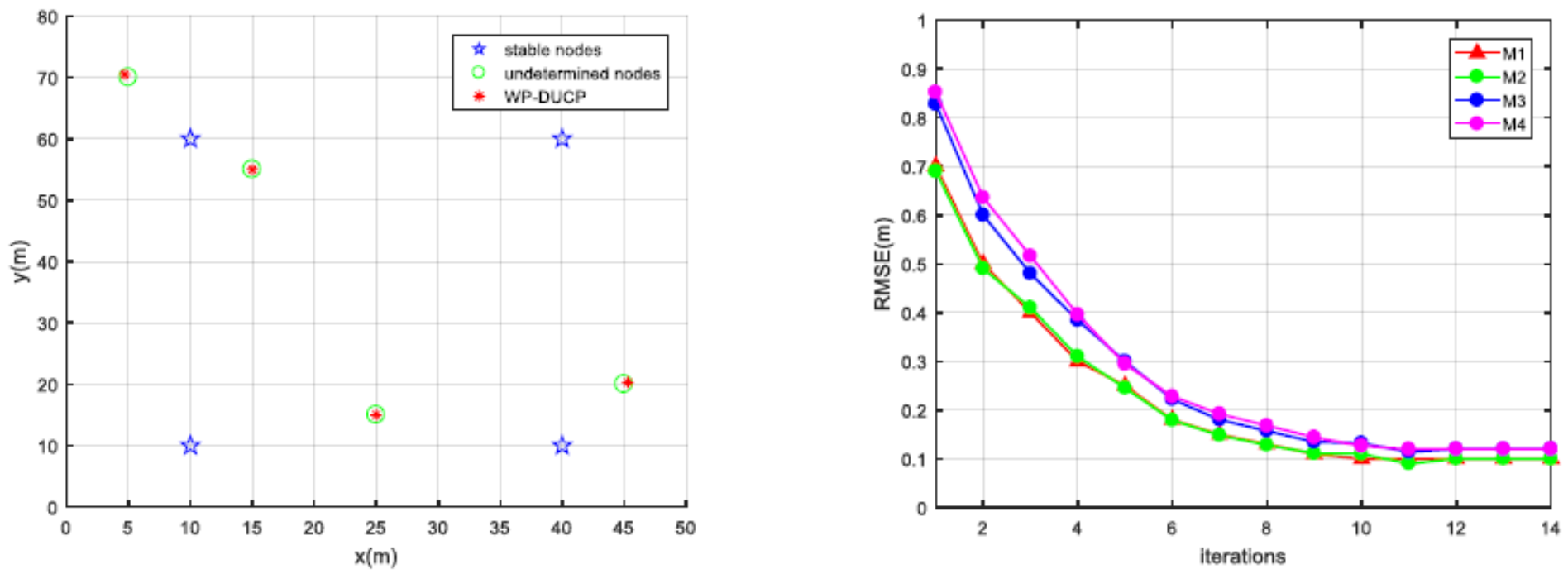

Figure 15

Co-location results of Scenario 2 (a) Position Estimation Result (b) RMSE vs Iteration

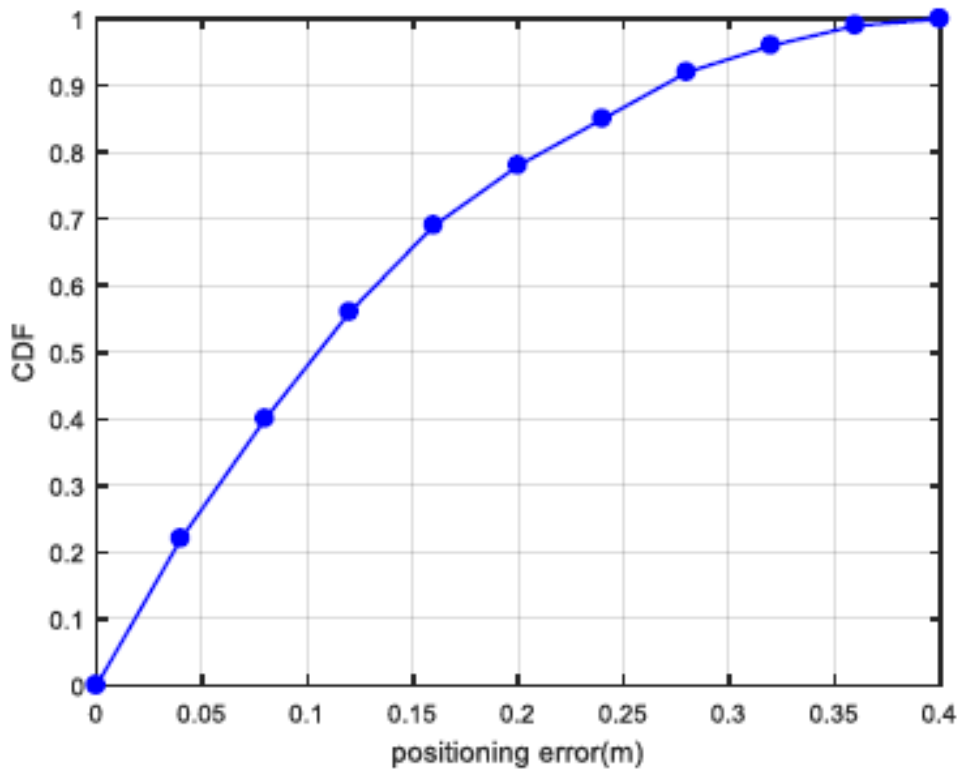

Figure 16

Positioning performance of WP-DUCP algorithm of Scenario 3 